\title{
Progressive methods for production process control
}

\author{
Olha Kolesnyk ${ }^{1}$, Peter Bubeník ${ }^{1}$, Juraj Čapek ${ }^{1}$ \\ ${ }_{1}$ University of Žilina, Faculty of Mechanical Engineering, Department of Industrial \\ Engineering \\ Univerzitná 8215/1, 01026 Žilina, Slovakia \\ olha.kolesnyk@fstroj.uniza.sk \\ peter.bubeni@fstroj.uniza.sk \\ juraj.capek@fstroj.uniza.sk
}

\begin{abstract}
Annotation: The aim of this paper is to provide an overview of algorithms, calculations, learning that support the latest advances in process control and operations in modern manufacturing. The aim of this review is not only to offer interested readers information on the latest state of control in production methods and applications in production, but also to provide scientists and experts with a vision of where the future is heading.
\end{abstract}

\section{Progressive methods}

Dramatic progress and the adoption of computing power, communication technologies and advanced, ubiquitous scanning have affected all aspects of modern manufacturing. In addition, as the company examines, the $4^{\text {th }}$ Industrial Revolution is characterized by access to and use of knowledge in the manufacturing enterprise, the very nature of production is evolving rapidly, with new, more complex processes and radically new products emerging in industries and academia. As for traditional production processes, they are also undergoing transformations in the sense that they face ever-increasing demands for quality, reliability, and productivity, needs that are addressed in the field of knowledge. Finally, in all production, we see the need to understand and control the interactions between the various stages of any given process, as well as the interactions between multiple products produced in the production system. All these factors motivated tremendous advances in the methodologies and applications of control theory in all aspects of production: at the level of process and equipment, at the level of production systems and operations.

Unlike automatic feedback control on sensed signals, the process input adjustment approach to predicted process responses is basically referred to as model-based process control [1]. Popular control strategies in this regard include adaptive process control, predictive model control, timedelayed control, long-term process control, etc.

The use of advanced algorithms and calculations is currently required for the implementation of progressive dilution methods. 
Therefore, it is appropriate to understand the principles of artificial intelligence, which allow intelligent behaviour, either in natural or artificial systems. Thus, the scientific purpose is to understand how to enable intelligent behaviour. The methodology is to design, create and experiment with computer systems that perform tasks commonly perceived as intelligent.

Progressive management methods can be divided into two groups of bioinspiring methods and machine learning.

\subsection{Bio-inspiring management methods}

Examples of Bio Inspirational Management Methods are described below.

\subsubsection{Evolutionary Computation}

One of the subgroups of artificial intelligence is evolutionary calculations. This is a special part of the calculations that draws inspiration from the process of evolution in nature. The power of evolution in nature can be seen in the diversity of species that make up our world. The basic characteristic of evolutionary calculations is the connection of the power of evolution in nature with a certain style of problem solving, namely by trial and error [2].

The development of automated problem solvers (algorithms) is one of the central themes of mathematics and computer science. The development of computers brings with it a rapidly growing demand for automated problem solving. However, the rate of development and research does not correspond to the rate of growth in demand. Therefore, the time available for problem analysis and algorithm development is constantly decreasing, while the complexity of the problems to be solved is increasing. Algorithms can be used for a wide range of problems and do not require much customization, while providing good (not necessarily optimal) solutions in a reasonable amount of time. Evolutionary algorithms fulfil all this and are therefore the answer to the challenge of automated solution of a wide range of problems in an ever-shorter time.

An example of the successful use of evolutionary algorithms for optimization is the schedule of university rooms and teaching. The main task of optimization is to reduce the number of collisions, either when one student should be in two places at the same time, or two different lectures take place at the same time in the same room. Creating a workable schedule (one that does not contain collisions) is not an easy task, as most of the time in all schedules is filled with unfeasible solutions. In addition, it is possible to create schedules to satisfy users and take into account, for example, that a student does not want to give more than two lectures, or to give lectures so that one day a week is free for self-study. On the other hand, there are demands from the university management to make the most of it, or to limit transfers around / between buildings. 


\subsubsection{Genetic Algorithms}

Genetic algorithms represent a classic group of methods in the field of evolutionary computations and were also historically the first seriously studied and developed group of algorithms. Genetic algorithms have nothing to do with genetic engineering or the creation of artificial organisms and the cloning of animals.

Genetic algorithms work by first creating an initial population with a certain number of chromosomes. This population may be generated randomly or may be intentionally generated in the expected optimal solution. Everyone in this population is evaluated using an evaluation function. Then this population is changed by genetic operators until the process is completed. Using the selection operator, we select a pair of chromosomes from a population. The probability of selection is related to the quality of the individuals. A specific individual may be selected more than once. This is followed by the crossing of selected chromosomes at a certain point and with a certain probability. Theoretically, the result of crossing can also be individuals identical to their parents. With a certain probability, a mutation is made on the resulting chromosomes, and they are then placed in the so-called the following population. The selection and selection steps are then repeated until the next population contains the same number of individuals as the original. At this point, the original population is replaced by the following. This process, which is repeated, is called the epoch of population evolution (one generation) and represents the three steps of selection, crossing and mutation. The number of repetitions of this process, i.e., the number of generations, can be given by the termination condition, e.g., number of generations, but also the required value [3].

Kríženie

Mutácia

A

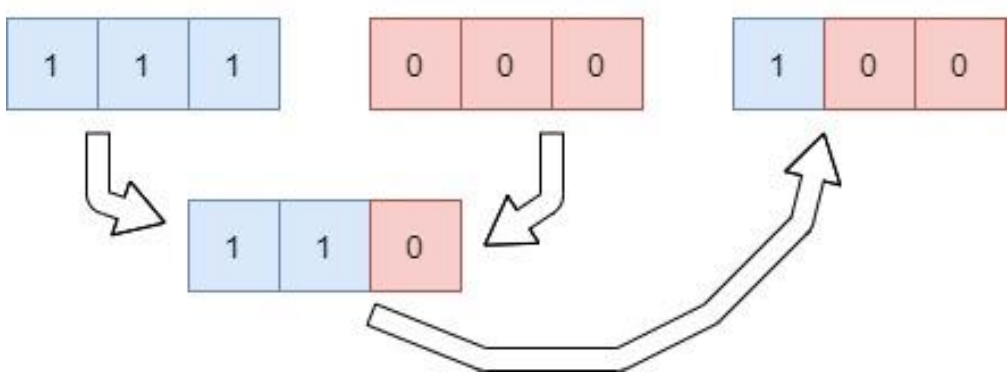

Figure 1 - Genetic operators of crossing and mutation.

The result of this cycle is usually one or more high quality chromosomes, which represent the optimal solution to the investigated problem. Since chance has a large effect on each experiment, no two experiments with a random initial distribution usually produce the same results. It is advisable to display various statistics, e.g., the best achieved individual of all generations, the generation in which he occurred across multiple trials. If the best solutions do not differ much from each other, it means that this solution is really optimal, we determined the local optimum quite accurately. 


\subsubsection{Ant algorithms}

Ant algorithms are inspired by the process that ants use to search for food (finding the shortest path between a food source and an anthill). The principle of indirect communication between ants is first a random search of the immediate area - searching for food near the anthill. During this activity, ants release chemicals into the ground, the so-called pheromones, which, however, disappear over time. All ants feel these substances and the consequence of this is the probability of choosing their other pathways, which is given by the actual concentrations of pheromones at the beginning of other possible pathways. This means that a higher concentration of pheromones represents a more efficient path between two points, and ants will prefer this [3].

\subsubsection{Ant colony optimization}

At the beginning of the algorithm, the various parameters with which the algorithm works (described in the following subsections) are initialized, pheromone traces. The algorithm then works until the termination condition is met - either the final number of iterations can be specified, or the achievement of an optimal solution or a solution that is usable with respect to the difficulty of the task.

\subsection{Machine learning}

It represents the ability of computers to observe the solution of tasks from a large amount of data. In a sense, this puts the use of computers upside down: until recently, when a programmer told a computer program step by step what to do, today the computer writes its own program. It can do this by finding various contexts, schemes or templates in the data and deriving a solution from them.

In this way, Al can gain different knowledge, make different predictions, or perform specific activities. Machine learning algorithms can be taught in three ways. In "supervised learning", training data includes input data, such as elapsed weather data, as well as the desired outcome, such as historical ice cream sales data [4].

This way it is also possible to teach him to classify data. For example, known data is divided into two categories $A$ and $B$, the task of the computer is to find the function of the decision boundary between the categories so that it can tell which new category it belongs to for all new data.

In "unsupervised learning", the machine receives only one group of data and must find connections in it. Then he can come up with some "wisdom" himself, for example to observe an anomaly in the data that we did not describe to him in advance. This can be used, for example, to find a hacker intrusion in many computer server security logs [4]. 
And "reinforcement learning" works in such a way that if a machine achieves a certain goal, it receives a "reward", and if it does not, a "punishment" awaits it. It's like a small child who got a TV remote control in his hand. If pressing different buttons leads to an undesirable result (screen off), the child will be dissatisfied, but if he finds the right button and the TV lights up, he will be happy, remember it and continue to learn.

Machine learning techniques have been known for a long time, but their recent rise has led to an increase in computer performance and the amount of data available. Data is especially needed for machine learning. Important activities supporting Al research include open data initiatives at the state, city, and corporate levels [5].

\section{Use of bio-inspiring methods}

The bio-inspiring algorithms are from a group of stochastic (a group of searching algorithms), an evolution of no-go algorithms. However, it is a heuristic algorithm. Evolutionary algorithms are search algorithms that try to solve a problem so that they know as little about it as possible. They take motivation from genetics and evolution. Their great advantage lies in the fact that they are simple. Thanks to which they are easy to implement and use in practice. They are one of the best tools for finding solutions to complex problems (e.g., from the NP complexity class).

This algorithm is based on a multiagent system. The population in this system consists of a collective - a colony (set of simple agents). The system is centralized and self-organizing. The population behaves collectively, which is a consequence of an indirect form of communication (using pheromone). The colony solves a task that is far beyond the capabilities of its members.

These algorithms can be used to solve problems otherwise difficult to solve, e.g., when interactions between individual parts are difficult to describable and the pros usually do not end at local maximum or always provide some solution or are easily implementable and parallelable.

Algorithms can be used for a variety of purposes, for example:

- Learning neural networks.

- Optimization of product loading and storage.

- Learning the business of robots.

- Design of the arrangement of production halls.

- Different planning problems (e.g., when individual tasks are interdependent). 


\section{Use of Machine Learning}

Practically, these machine learning algorithms can be applied in different areas. They are most often used in discovering knowledge in databases. Useful knowledge can be found in the analysis of the shopping cart, it is possible to conduct surveys of the purchase - the ability of customers, their preferences in the selection of products (goods, insurance, ...) etc. Machine learning algorithms can also be applied in every other area where it is a solution of classification task: processing text documents, detecting suspicious banking operations, predicting the development of sheet metal defects during rolling and so on.

\section{Conclusion}

The aim of this paper was to provide an overview of algorithms, calculations, learning that support the latest advances in process control and operations in modern manufacturing, as well as the use of algorithms, calculations, learning in practice to streamline production dilution processes.

\section{Acknowledgment}

This work was supported by the VEGA 1/0248/21 - Research of innovative solutions for segmentation and sustainability of product regarding assembly operations.

\section{References}

[1] DJURDJANOVIC, D.; MEARS, L.; NIAKI, F.A.; HAQ, A.U.; LI, L. Process and operations control in modern manufacturing. In Proceedings of the 12th International Manufacturing Science and Engineering Conference, Los Angeles, CA, USA, 4-8 June 2017.

[2] EIBEN, AGOSTON E, SMITH, JAMES E, Introduction to Evolutionary Computing, Berlin : Springer, 2003, ISBN 3-540-40184-9

[3] MITCHELL, M. An Introduction to Genetic Algorithms, Cambridge: MIT Press, 1998. 209 s. ISBN 0-262-63185-7

[4] GREGOR, M., GREGOR, T., PATKA, J. 2016 d. Továrne budúcnosti a pokrokové technológie. Produktivita a inovácie. No. 4, s. 22-25, 2016, ISSN1339 2271

[5] IBM researchers check Al bias with counterfactual text. Dostupné na internete < www.ibm.com/blogs/research/2019/12/2020-ai-predictions/> 